\title{
Lack of visual field asymmetries for spatial cueing in reading parafoveal Chinese characters
}

\author{
Chunming Luo ${ }^{1} \cdot$ Roberto Dell'Acqua $^{2} \cdot$ Robert W. Proctor ${ }^{3} \cdot{\text { Xingshan } \text { Li }^{1}}^{1}$
}

Published online: 13 May 2015

(C) Psychonomic Society, Inc. 2015

\begin{abstract}
In two experiments, we investigated whether visual field (VF) asymmetries of spatial cueing are involved in reading parafoveal Chinese characters. These characters are different from linearly arranged alphabetic words in that they are logograms that are confined to a constant, square-shaped area and are composed of only a few radicals. We observed a cueing effect, but it did not vary with the VF in which the Chinese character was presented, regardless of whether the cue validity (the ratio of validly to invalidly cued targets) was 1:1 or 7:3. These results suggest that VF asymmetries of spatial cueing do not affect the reading of parafoveal Chinese characters, contrary to the reading of alphabetic words. The mechanisms of spatial attention in reading parafoveal English-like words and Chinese characters are discussed.
\end{abstract}

Keywords Attention $\cdot$ Parafoveal processing $\cdot$ Chinese characters

A well-established finding in the reading literature is that participants respond more quickly and accurately to written English-like words presented in the right visual field (RVF) than in the left visual field (LVF; see, e.g., Ellis, 2004; Nicholls \& Wood, 1998). This RVF advantage for words

Chunming Luo

luocm@psych.ac.cn

1 Key Laboratory of Behavioral Science, Institute of Psychology, Chinese Academy of Sciences, 16 Lincui Road, Chaoyang District, Beijing 100101, China

2 Centre for Cognitive Neuroscience, University of Padua, Padua, Italy

3 Department of Psychological Sciences, Purdue University, West Lafayette, IN, USA usually has been ascribed to RVF words benefiting from direct access to the left cerebral hemisphere (LH), which is specialized for language (the structural hypothesis; Kimura, 1961). For example, Hunter and Brysbaert (2008) found that participants who exhibited a clear RVF advantage in picture-naming and word-naming tasks were LH dominant for language, whereas participants with an LVF advantage or no VF asymmetry showed bilateral or right-cerebral dominance for language.

However, two popular alternative interpretations of VF asymmetries, particularly relevant for the present study, are concerned with the manner in which spatial attention can be allocated across the VF (Kinsbourne, 1970; McCann, Folk, \& Johnston, 1992; Mondor \& Bryden, 1992; Nicholls \& Wood, 1998). The attentional-bias account (Kinsbourne, 1970) posits that VF asymmetries are the result of an imbalance in activation between the hemispheres. In a resting state, the hemispheres are in a state of reciprocal inhibitory balance, resulting in an even allocation of attention between the LVF and RVF. However, when one hemisphere is activated by the act, or the expectation, of processing certain classes of information, attentional resources will be subsequently deployed to the contralateral hemispace. The presentation of a word would result in an activation of the $\mathrm{LH}$, which in turn would cause a bias of attentional resources to the RVF and facilitate word processing in that VF. Attentional bias likely arises from the scanning habits developed during the process of learning to read. Mishkin and Forgays (1952) observed the RVF advantage for English words, but an LVF advantage for Yiddish words (a right-to-left script). However, Faust, Kravetz, and Babkoff (1993) also found an RVF advantage for Hebrew words (a right-to-left script).

The attentional-advantage account (Mondor \& Bryden, 1992), another explanation of the VF asymmetry, posits that different word processing styles are used by the left and right 
(RH) hemispheres. The LH can process words when few attentional resources are allocated to the task, whereas the $\mathrm{RH}$ requires more attentional resources for the same task. The RVF advantage for words is assumed to occur because processing RVF words is automatic and requires little or no attention, whereas processing LVF words is sequential and requires attention (Mondor \& Bryden, 1992; Nicholls \& Wood, 1998). This account is similar to the explanation of the greater impact of word length on recognizing visual words in the LVF than in the RVF (Bouma, 1973; Ellis, 2004; Ellis, Young, \& Anderson, 1988). The asymmetry of the word length effect has been attributed to different modes of lexical access, with efficient, parallel processing of letters in the LH and nonparallel processing in the RH (Ellis et al., 1988).

Studies using the spatial-cueing paradigm and visual halffield presentation have demonstrated a larger cueing effect (difference between a condition in which the cue is in the same location as the word and a condition in which the cue is in the opposite location) for LVF than for RVF words (Ducrot \& Grainger, 2007; Gatheron \& Siéroff, 1999; Mondor \& Bryden, 1992; Nicholls \& Wood, 1998), and often no cueing effect at all for the RVF words (e.g., Mondor \& Bryden, 1992; Nicholls \& Wood, 1998). Both the attentional-bias and attentional-advantage hypotheses can explain the cueing effect asymmetry (Siéroff \& Riva, 2011), but only the latter can explain the lack of a cueing effect in the RVF.

To allow a full understanding of the rationale of the present experiment, it was based on the following premise: Chinese characters are different from linearly arranged alphabetic words, in that they are logograms composed of a varying number of strokes and are always confined to a constant square-shaped area. Modern Chinese writing is mainly divided into simple and complex characters. Simple characters occupy about $5 \%$ of all characters and have holistic visual patterns that cannot be divided meaningfully into sublexical units, such as 大 (big) and 马 (horse). Complex characters constitute about $95 \%$ of all characters and have two or more radicals. About $80 \%$ of complex characters are phonetic compounds, consisting of phonetic radicals that provide cues to the pronunciation of their host characters, and semantic radicals that usually imply the meaning of their host characters. For example, 妈 (“mother") is constructed from a phonetic radical 马 ("horse") and a semantic radical 女 ("female"). About $13 \%$ of complex characters are ideogrammic compounds constructed by combining two or three radicals' meanings, and these radicals are unrelated to the host character in pronunciation (e.g., Luo, Proctor, \& Weng, 2015; Luo, Proctor, Weng, \& Li, 2014). For instance, combining 日 ("sun”) and 月 ("moon”), the two natural sources of light, makes 明 (“bright”). In Chinese, words might consist of one or more characters, but the majority of words are made up of two characters, and each character has its own pronunciation.
Although English-like words show an RVF advantage, it is unclear whether an RVF advantage occurs when reading parafoveal Chinese characters. Some studies have revealed an RVF advantage (Besner, Daniel, \& Slade, 1982), whereas others have shown an LVF advantage (Cheng \& Yang, 1986; Tzeng, Hung, Cotton, \& Wang, 1979), and still others have demonstrated no such asymmetries (Fang, 1997). Moreover, it is unclear whether VF asymmetries of the spatial-cueing effect are involved in reading parafoveal Chinese characters.

As we reviewed above, attention plays a role in the VF asymmetry when reading English-like words (e.g., Ducrot \& Grainger, 2007; Gatheron \& Siéroff, 1999; Mondor \& Bryden, 1992). In the present study, we used the cueing paradigm and Chinese characters to investigate whether attention also plays a role in reading parafoveal Chinese characters, by manipulating cue validity and cueing. We anticipated that the results would discriminate the aforementioned attentional-bias and attentional-advantage accounts, because each Chinese character is composed of a few radicals and confined to a constant, square-shaped area, which is different from linearly arranged alphabetic words.

\section{Experiment 1}

In this experiment, the ratio of validly to invalidly cued targets was $1: 1$, and therefore the spatial cues were valid predictors of the target's location on half of the trials. The attentionaladvantage account predicts that there should be no VF difference and that the cueing effect should be invariant of VF, because each Chinese character is confined to a constant square-shaped area and is composed of only a few radicals, and thus need not be processed sequentially when it is presented in the LVF. The attentional-bias account predicts that more attentional resources would be allocated to the RVF than to the LVF, resulting in a VF difference and a larger cueing effect in the LVF than in the RVF.

\section{Method}

Participants A total of 28 participants, from 19 to 27 years of age (14 females, 14 males), took part in this experiment. All were right-handed, native speakers of Chinese (Putonghua) with normal or corrected-to-normal vision. They were given informed consent, were naive as to the purpose of the study, and received compensation for their participation.

Apparatus and stimuli The stimuli were presented in white on a super VGA high-resolution color monitor with a black background. A computer, running the E-Prime 1.1 software, controlled the presentation of the stimuli, timing operations, and data collection. Participants viewed the monitor from a distance of $58 \mathrm{~cm}$ in a dimly lit room. 
Two-hundred character pairs were used as the stimuli in two conditions (100 pairs for each condition). In the character condition, the central and target characters were two true characters in the Chinese lexicon, but they did not compose a word in contemporary Chinese, which is usually read from left to right horizontally. In the noncharacter condition, the central character was also a true Chinese character, but the target was a nonexistent character constructed from a number of randomly assembled Chinese strokes that were equivalent, on average, to the number of strokes composing a true Chinese character. The mean numbers of strokes were 8.3 and 8.4 for true characters and noncharacters, respectively. The average frequencies of the central characters were 778 and 787 per million for the character and noncharacter conditions, respectively. The average frequencies of the targets, based on the Modern Chinese Frequency Dictionary (Beijing Language Institute, 1985), were 998 per million for the character condition (ranged from 7 to 9,458) with 2.42 radicals on average (ranged from 1 to 5). Each character displayed on a given trial was always of the same size and subtended $1.5^{\circ} \times 1.5^{\circ}$. The central and target characters of each pair were not related in semantics, phonology, or orthography.

Procedure and design Each trial proceeded as follows (see Fig. 1): A fixation cross appeared in the center of the screen for $1 \mathrm{~s}$, and then was replaced by a Chinese character for $500 \mathrm{~ms}$. After that, a rectangular cue $\left(1.6^{\circ} \times 1.6^{\circ}\right)$ appeared (approximately $5.5^{\circ}$ ) to either the left or the right of the fixation cross for $100 \mathrm{~ms}$. The target character (or noncharacter) then was presented for $150 \mathrm{~ms}$ in the same location occupied by the cue or in the opposite location. Finally, the screen became blank for $1.5 \mathrm{~s}$ or until the participant responded. The intertrial interval was $1 \mathrm{~s}$. The central character was used to activate the language-processing system prior to the occurrence of the cue, and it was not related to the target in semantics, phonology, or orthography.

Participants were instructed to discriminate, as quickly and accurately as possible while not moving their eyes, whether the target was a character or noncharacter by pressing the "V" or the " $\mathrm{M}$ " key with their left or right index fingers, respectively. The mapping of the response hand to the stimulus type was counterbalanced across participants. The experiment had a 2 (VF: LVF, RVF) $\times 2$ (Target Type: character, noncharacter) $\times 2$ (Cueing: valid, invalid) design, with 30 observations in each condition, and all of the factors were between-items manipulations.

\section{Results}

Only the trials on which the targets were Chinese characters were included in the following analyses of variance (ANOVAs), by subjects $\left(F_{1}\right)$ and items $\left(F_{2}\right)$. A total of $3.83 \%$ of the trials were excluded due to erroneous behavior, $0.43 \%$ due to excessively slow responses $(>1$, $500 \mathrm{~ms}), 2.30 \%$ due to incorrect responses, and $1.10 \%$ because the response times (RTs) were more than three standard deviations from the mean for each condition and each participant. The mean correct RTs and percentages of errors (PEs) for each condition are presented in Table 1.

The analysis of RTs revealed significant main effects of cueing, $F_{1}(1,27)=74.47, M S E=3,039, p<.001, \eta_{\mathrm{p}}{ }^{2}=$ $.734 ; F_{2}(1,96)=74.53, M S E=2,541, p<.001, \eta_{\mathrm{p}}{ }^{2}=.437$, and of VF, $F_{1}(1,27)=6.88, M S E=2,159, p=.014$, $\eta_{\mathrm{p}}^{2}=.203 ; F_{2}(1,96)=4.10, M S E=2,541, p=.046$, $\eta_{\mathrm{p}}{ }^{2}=.041$, with faster responses for valid than for invalid cues and for the RVF than for the LVF. The interaction between VF and cueing was not reliable, $F_{1}$ and $F_{2}<1$.

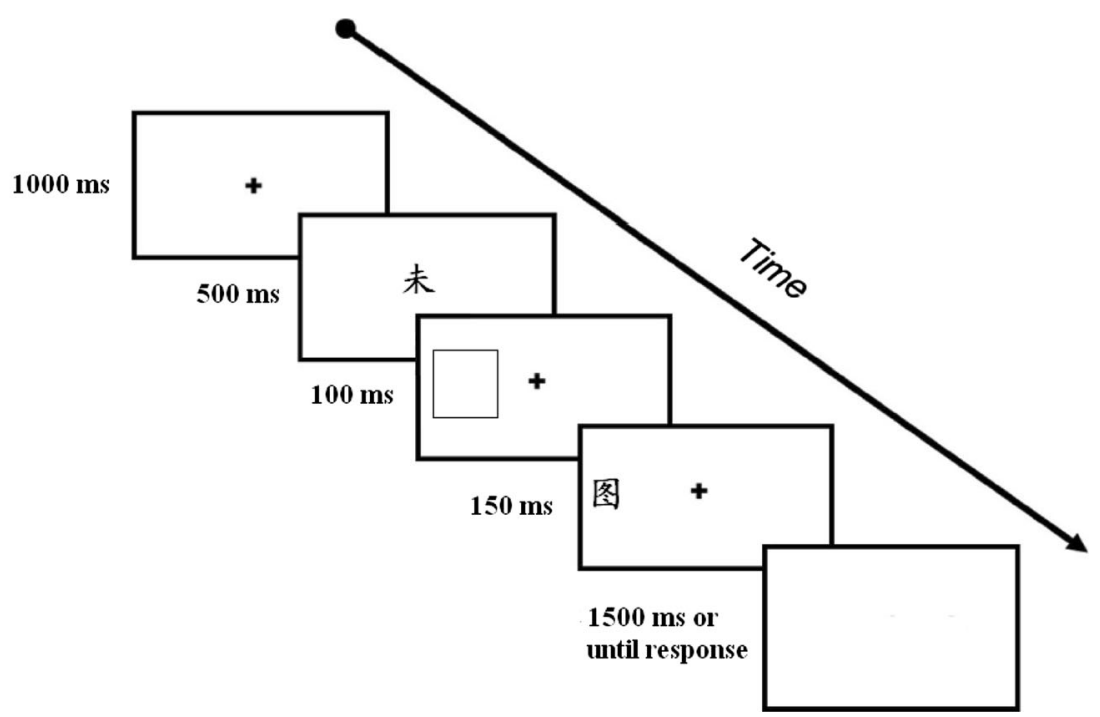

Fig. 1 Example of a typical trial on which the target is a Chinese character 
Table 1 Mean response times (RTs, in milliseconds), percentages of error (PEs), and standard deviations (in parentheses) as a function of target location and cueing condition in Exp. 1 (cue validity $=.5$ ) and Exp. 2 (cue validity $=.7$ )

\begin{tabular}{|c|c|c|c|c|c|c|c|}
\hline & & \multicolumn{3}{|c|}{ Left Visual Field } & \multicolumn{3}{|c|}{ Right Visual Field } \\
\hline & & Valid & Invalid & $\mathrm{CE}$ & Valid & Invalid & $\mathrm{CE}$ \\
\hline \multirow[t]{2}{*}{ Exp. 1} & RTs & $639(167)$ & $731(181)$ & 92 & $618(151)$ & $707(181)$ & 89 \\
\hline & PEs & $4.3(0.20)$ & $4.9(0.22)$ & 0.6 & $3.4(0.18)$ & $2.7(0.16)$ & -0.7 \\
\hline \multirow[t]{2}{*}{ Exp. 2} & RTs & $605(157)$ & $687(155)$ & 82 & $597(157)$ & $681(151)$ & 84 \\
\hline & PEs & $1.9(0.14)$ & $1.7(0.13)$ & -0.2 & $2.1(0.14)$ & $0.9(0.10)$ & -1.2 \\
\hline
\end{tabular}

Cueing effect $(\mathrm{CE})=$ Invalid - Valid

PEs showed a main effect of VF in the analyses by subjects, $F_{1}(1,27)=6.09, M S E=0.001, p=.020, \eta_{\mathrm{p}}{ }^{2}=.184$, but not by items, $F_{2}(1,96)=1.81, M S E=0.002, p=.181, \eta_{\mathrm{p}}{ }^{2}=.019$. The main effect of cueing was not significant, $F_{1}$ and $F_{2}<1$, nor was the interaction between VF and cueing, $F_{1}$ and $F_{2}<1$.

\section{Discussion}

As had been observed in some previous studies (Besner et al., 1982), the results showed an RVF advantage for character targets and a cueing effect of $91 \mathrm{~ms}$, indicating that the cue manipulation directed attention to the validly cued location. The cueing effects did not vary with VF, suggesting that the role of attention in reading parafoveal Chinese characters may not have VF differences. The RVF advantage in RTs for characters was consistent with the prediction of the attentional-bias but not of the attentional-advantage account. By contrast, that the cueing effect was invariant of VF was consistent with the prediction of the attentional-advantage but not the attentionalbias account.

\section{Experiment 2}

In this experiment, the ratio of validly to invalidly cued targets was $7: 3$, so that the spatial cues were valid predictors of the targets' location on $70 \%$ of the trials. These highly predictive cues likely encouraged participants to attend to them voluntarily and to associate the appearance of the target with the cued location, which might affect the attentional bias. Therefore, the attentional-bias account predicted that the VF differences in responses and in the cueing effect should be reduced or eliminated. The predictions of the attentionaladvantage account were the same as in Experiment 1.

\section{Method}

Participants A total of 28 participants from 19 to 29 years of age (14 females, 14 males) took part in this experiment. All had normal or corrected-to-normal vision and were naive to the purpose of the experiment.

Apparatus, stimuli, procedure, and design These were the same as in Experiment 1, except that the number of validly cued versus invalidly cued trials was 7:3, with 36 character trials and 36 noncharacter trials for the invalidly cued condition.

\section{Results}

Only the trials in which the targets were Chinese characters were included in the following ANOVAs by subjects $\left(F_{1}\right)$ and items $\left(F_{2}\right)$. A total of $1.65 \%$ of the trials were excluded from the RT analysis due to erroneous behavior, $0.28 \%$ due to excessively slow responses ( $>1,500 \mathrm{~ms}), 0.5 \%$ for RTs more than three standard deviations from the mean for each condition and each participant, and $0.87 \%$ due to incorrect responses. The mean correct RTs and PEs for each condition are presented in Table 1.

The analyses of RTs showed a main effect of cueing, $F_{1}(1,27)=104.15, M S E=1,868, p<.001, \eta_{\mathrm{p}}{ }^{2}=.794$; $F_{2}(1,96)=25.39, M S E=3,549, p<.001, \eta_{\mathrm{p}}^{2}=.209$. The main effect of VF was not significant, $F_{1}(1,27)=1.29$, $M S E=1,031, p=.265, \eta_{\mathrm{p}}{ }^{2}=.046 ; F_{2}<1$, nor was the interaction between VF and cueing, $F_{1}$ and $F_{2}<1$.

The analyses of PEs showed no main effect of VF, $F_{1}<1$; $F_{2}(1,96)=1.19, M S E=.003, p=.278, \eta_{\mathrm{p}}{ }^{2}=.012$, or of cueing, $F_{1}(1,27)=2.05, M S E=.001, p=.164, \eta_{\mathrm{p}}{ }^{2}=.070$; $F_{2}<1$. The interaction between VF and cueing was also not significant, $F_{1}$ and $F_{2}<1$.

\section{Discussion}

As in Experiment 1, we observed a cueing effect of $83 \mathrm{~ms}$ on RTs, indicating that the cue manipulation directed attention to the cued location. The cueing effects did not vary with VF, suggesting that the role of attention in reading parafoveal Chinese characters may not have VF differences. This result is compatible with the prediction of the attentional-advantage account, but not with the attentional-bias account. The lack of a VF difference for recognizing character targets, on the other hand, was consistent with the prediction of the attentional-bias but not of the attentional-advantage account.

\section{General discussion}

In the present study, we investigated whether VF asymmetries of spatial cueing are involved in reading parafoveal Chinese characters and whether such results can be explained by an attentional-advantage or an attentional-bias account. In Experiments 1 and 2, when the cue validity was either 1:1 or 
$7: 3$, we found a reliable cueing effect that did not vary with $\mathrm{VF}$, suggesting that no VF asymmetries of spatial cueing effect are involved in reading parafoveal Chinese characters.

The attentional-advantage account posits that different word-processing styles are used by the LH and RH. Processing RVF words by the LH may be automatic and require little or no attention, whereas processing LVF words by the RH may be sequential and require attention (Mondor \& Bryden, 1992; Nicholls \& Wood, 1998). Therefore, this account can explain the absence of VF asymmetries for the spatial-cueing effect, because each Chinese character was confined to a constant square-shaped area and had only a few radicals, and thus did not need to be processed sequentially when it was presented in the LVF. By contrast, the attentionalbias account (Kinsbourne, 1970) posits that a linguistic task activates the LH and makes it control behavior and attention, resulting in more attentional resources being allocated to the RVF than to the LVF, thus producing an RVF advantage. Therefore, this account cannot easily explain the lack of VF asymmetries in the spatial-cueing effect, because it would predict that more attentional resources should be allocated to the RVF than to the LVF, resulting in a greater cueing effect in the LVF than in the RVF.

Moreover, we observed an RVF advantage in recognizing the characters in Experiment 1, but no VF difference in Experiment 2. This result can be explained by the attentional-bias account, which would assume that more attentional resources were allocated to the RVF than to the LVF in Experiment 1, in which the cue validity was 1:1. However, in Experiment 2, in which the cue validity was $7: 3$, the highly predictive cue likely encouraged observers to voluntarily attend to them and to associate the appearance of the target with the validly cued location, which could have changed the allocation of attention resources in the VF. The attentionaladvantage account can explain the result in Experiment 2 but not that in Experiment 1, because each Chinese character was confined to a constant square-shaped area, and thus did not need to be sequentially processed when it was presented in the LVF.

Why are VF asymmetries of spatial cueing a feature of reading English-like words but not of reading parafoveal Chinese characters? This difference may be present because alphabetic words are linearly arranged and consist of many letters, whereas each Chinese character is confined to a constant square-shaped area and the majority of characters are either phonetic $(80 \%)$ or ideogrammic $(13 \%)$ compounds composed of two or three radicals. Each character has few radicals and is confined to a constant square-shaped area, resulting in LVF Chinese characters being recognized very quickly and not being processed sequentially. These speculations are in parallel with the findings in some recent case studies, which have suggested no evidence for the radicalby-radical reading strategy in Chinese pure alexia or for a linear relationship between the number of radicals in a character and the time taken to read it (Chen et al., 2014; Shan, Zhu, Xu, Luo, \& Weng, 2010; but see Yin \& Butterworth, 1998), as compared to the letter-by-letter reading strategy in alphabetic languages.

By contrast, English-like words are linearly arranged and composed of more letters, which may cause LVF words to be recognized slowly and to need to be processed sequentially. Consequently, recognizing long words is less difficult in the RVF than in the LVF (Ellis, 2004; Siéroff, Dahmen, \& Fagard, 2012). Moreover, previous studies have also shown a larger effect of word length on recognition in the LVF than in the RVF (Ellis, 2004; Lavidor \& Ellis, 2002) and a cueing effect obtained with words when word length was increased to ten letters (Auclair \& Siéroff, 2002). Therefore, this attentional component involved in reading English-like words may occur because word length has different effects when reading LVF and RVF words.

In the present study, the targets were Chinese characters whose average frequency was 998 per million (ranging from 7 to 9,485 ). Such characters can be used as one-character words in Chinese, including the lowest-frequency characters, 羡 (“admire," frequency =7), 肖 ("resemble," frequency =9), and 橡 ("oak," frequency =12). However, these characters also can be combined with other characters to construct other words. That the stimuli were meaningful rules out an explanation through which the VF asymmetries of spatial cueing in reading English-like words, but not parafoveal Chinese characters, may be due to the English words carrying meaning, where Chinese characters do not.

In conclusion, VF asymmetries can vary with cue validity when reading parafoveal Chinese characters, but there are no $\mathrm{VF}$ asymmetries of spatial cueing in this process, suggesting that cueing is not a determinant of VF asymmetries. The VF asymmetries of cueing observed in reading parafoveal English-like words may occur because the words are linearly arranged and composed of more letters, which may cause LVF words to be recognized slowly and processed sequentially.

Author note This research was supported in part by grants from the National Science Foundation of China (No. 31470984) and from the National Social Science Foundation of China (No. 11AZD119).

\section{References}

Auclair, L., \& Siéroff, E. (2002). Attentional cueing effect in the identification of words and pseudowords of different length. Quarterly Journal of Experimental Psychology, 55A, 445-463.

Besner, D., Daniel, S., \& Slade, C. (1982). Ideogram reading and right hemisphere language. British Journal of Psychology, 73, 21-28.

Bouma, H. (1973). Visual interference in the parafoveal recognition of initial and final letters of words. Vision Research, 13, 767-782.

Chen, Z., Shao, H., Xu, M., Peng, G., Liu, P., Liu, X., ... Luo, B. (2014). Is radical-by-radical reading strategy effective for Chinese pure 
alexia? A case study. Neuropsychology, 29, 402-408. doi:10.1037/ neu0000130

Cheng, C. M., \& Yang, M. J. (1986). Lateralization in the visual perception of Chinese characters and words. Brain and Language, 36 , 669-689.

Ducrot, S., \& Grainger, J. (2007). Deployment of spatial attention to words in central and peripheral vision. Perception \& Psychophysics, 69, 578-590. doi:10.3758/BF03193915

Ellis, A. W. (2004). Length, formats, neighbors, hemispheres, and the processing of words presented laterally or at fixation. Brain and Language, 88, 355-366.

Ellis, A. W., Young, A. W., \& Anderson, C. (1988). Modes of word recognition in the left and right cerebral hemispheres. Brain and Language, 35, 254-273.

Fang, S.-P. (1997). Morphological properties and the Chinese characterword difference in laterality patterns. Journal of Experimental Psychology: Human Perception and Performance, 23, 1439-1453. doi:10.1037/0096-1523.23.5.1439

Faust, M., Kravetz, S., \& Babkoff, H. (1993). Hemispheric specialization or reading habits: Evidence from lexical decision research with Hebrew words and sentences. Brain and Language, 44, 254-263.

Gatheron, D., \& Siéroff, E. (1999). Right hemifield superiority in reading and attentional factors. Brain and Cognition, 40, $122-125$.

Hunter, Z. R., \& Brysbaert, M. (2008). Visual half-field experiments are a good measure of cerebral language dominance if used properly: Evidence from fMRI. Neuropsychologia, 46, 316-325. doi: 10.1016/j.neuropsychologia.2007.07.007

Institute, B. L. (1985). Modern Chinese frequency dictionary. Beijing: Beijing Language Institute Press.

Kimura, D. (1961). Cerebral dominance and the perception of verbal stimuli. Canadian Journal of Psychology, 15, 166-171.

Kinsbourne, M. (1970). The cerebral basis of lateral asymmetries in attention. Acta Psychologica, 33, 193-201.
Lavidor, M., \& Ellis, A. W. (2002). Word length and orthographic neighborhood size effects in the left and right cerebral hemispheres. Brain and Language, 80, 45-62.

Luo, C., Proctor, R. W., Weng, X., \& Li, X. (2014). Spatial Stroop interference occurs in the processing of radicals of ideogrammic compounds. Psychonomic Bulletin \& Review, 21, 715-720. doi:10.3758/ s13423-013-0533-x

Luo, C., Proctor, R. W., \& Weng, X. (2015). A Stroop effect emerges in the processing of complex Chinese characters that contain a colorrelated radical. Psychological Research, 79, 221-229. doi:10.1007/ s00426-014-0553-9

McCann, R. S., Folk, C. L., \& Johnston, J. C. (1992). The role of spatial attention in visual word processing. Journal of Experimental Psychology: Human Perception and Performance, 18, 1015-1029. doi:10.1037/0096-1523.18.4.1015

Mishkin, M., \& Forgays, D. G. (1952). Word recognition as a function of retinal locus. Journal of Experimental Psychology, 43, 43-48.

Mondor, T. A., \& Bryden, M. P. (1992). On the relation between visual spatial attention and visual field asymmetries. Quarterly Journal of Experimental Psychology, 44A, 529-555.

Nicholls, M. E. R., \& Wood, A. G. (1998). The contribution of attention to the right visual field advantage for word recognition. Brain and Cognition, 38, 339-357.

Shan, C. L., Zhu, R. J., Xu, M. W., Luo, B. Y., \& Weng, X. C. (2010). Implicit reading in Chinese pure alexia. Brain and Language, 114, 147-156.

Siéroff, E., Dahmen, R., \& Fagard, J. (2012). Mechanisms of attention in reading parafoveal words: across-linguistic study in children. Neuropsychology, 26, 334-346. doi:10.1037/a0027572

Siéroff, E., \& Riva, M. (2011). Attention and the identification of parafoveal words in school-age children and adults. Journal of Neurolinguistics, 24, 420-434.

Tzeng, O. J. L., Hung, D. L., Cotton, B., \& Wang, S. Y. (1979). Visual lateralization in reading Chinese characters. Nature, 382, 499-501.

Yin, W. G., \& Butterworth, B. (1998). Chinese pure alexia. Aphasiology, $12,65-76$. 